\title{
Probing inhomogeneous diffusion in the microenvironments of phase-separated polymers under confinement
}

\author{
Marjan Shayegan $^{1}$, Radin Tahvildari ${ }^{1}$, Lydia Kisley ${ }^{2}$, Kimberly Metera $^{1}$, Stephen W. Michnick ${ }^{3}$, and Sabrina R. Leslie ${ }^{1, \bowtie}$ \\ ${ }^{1}$ Department of Physics, McGill University, Montreal, Quebec, Canada H3A 2T8. Fax: +1 514398 8434; Tel: +1 514398 1835. Email: Sabrina.leslie@mcgill.ca \\ ${ }^{2}$ Beckman Institute for Advanced Science and Technology, University of Illinois at Urbana-Champaign, Urbana, Illinois, United States. \\ ${ }^{3}$ Département de Biochimie, Université de Montréal, Montréal, Québec, Canada.
}

\begin{abstract}
Biopolymer condensates formed by liquid-liquid phase separation of proteins and nucleic acids have been recently discovered to be prevalent in biology. These dynamic condensates behave like biochemical reaction vessels but little is known about their structural organization and properties. Their biophysical properties and catalytic functions are likely related to condensate size, and thus it is critical that we study them on scales found in vivo. However, previous in vitro studies of condensate assembly and physical properties have involved condensates up to 1000 times larger than those found in vivo. Here, we report the application of confinement microscopy to visualize condensates and control their sizes by creating appropriate confinement length scales relevant to the cell environment. We observe anomalous diffusion of probe particles embedded within confined condensates, as well as heterogeneous dynamics in condensates formed from PEG/dextran and in ribonucleoprotein complexes of RNA and the RNA-binding protein Dhh1. We propose that the non-Gaussian dynamics we observe may indicate a hopping diffusion mechanism inside condensates. We also observe that for dextran-rich condensates, but not for ribonucleo condensates, probe particle diffusion depends on condensate size.
\end{abstract}

Liquid-liquid phase separation is found throughout nature and has important consequences in biology (1-3). For example, biopolymer condensates, or non-membranous organelles (NMOs), are dynamic structures within the cyto- and nucleoplasm of cells that form via reversible and highly controlled liquid-liquid phase separation of proteins and nucleic acids. Inside cells, different NMOs perform a variety of functions (3-8): concentrating molecules to enhance biochemical reaction rates within the diverse and crowded cell interior; isolating catalytic processes; sequestering and storing materials for later release; and helping cells respond to stress. Some are further sub-compartmentalized and may generate gradients and chemical potentials (5). Some appear to concentrate specific molecules while simultaneously excluding others. Through aberrant formation, regulation, or stability, NMOs may also contribute to neurodegenerative conditions including Alzheimer's and Huntington's diseases. However, the principles governing NMO assembly, disassembly and functions remain unknown (9-12). Furthermore, the relationships between NMO size, properties, and functions need to be clar- ified. NMOs scale with the size of the cell $(4,13)$, and cell size dysregulation is connected to many diseases, including cancers (14).

Detailed biophysical studies of phase-separated biopolymer condensates will help clarify how these important structures function. Important factors for the phase separation of biopolymer condensates include: 1) the content of intrinsically disordered regions in the component proteins, and 2) interactions between folded protein or RNA domains with linear regions (15). The mechanical properties of the phase separated condensates (e.g. surface tension and viscoelastic properties) and the interactions among their component proteins and nucleic acids are expected to control condensate coalescence, internal structuring, diffusion within the condensate, and exchange of molecules with the cyto- or nucleoplasm, and thus must heavily influence condensate function (3). For example, it has been proposed that the viscoelastic properties of NMOs control enzymatic processes by lowering the flux of specific molecular species (16).

In vitro studies of biopolymer condensates allow for controlled conditions in which to study condensate assembly and properties, and have probed a range of parameters controlling phase separation (Refs.(3, 17-20)are just a few examples of recent reviews describing insights from in vitro studies). However, typical in vitro techniques cannot approach simulating the naturally crowded, complex, and confined conditions found in cells, and reconstituted condensates can vary greatly in size and be up to $10-1000$ times larger $(\sim 10-100$ $\mu \mathrm{m})$ than those observed in vivo $(\sim 100 \mathrm{~nm}$ for P-bodies $(7))$. To understand natural NMOs, it is crucial to determine how these vastly different size regimes might influence condensate biophysical properties.

Experimental approach: Convex Lens-induced Confinement (CLiC) fluorescence microscopy (21-24) is applied to control condensate size and monitor how size affects diffusion within them. In this technique, condensates are formed within tiny wells (450 $\mathrm{nm}$ deep and 3-50 $\mu \mathrm{m}$ in diameter) to limit condensate growth. In a typical confinement microscopy experiment, a fluid sample of biomolecules is loaded into a customdesigned imaging chamber made from two glass coverslips separated by a spacer. As shown in Fig. 1, the bottom coverslip (the chamber floor) contains an array of embedded wells with highly-controlled and well-defined nano- to microscale 
geometry. After sample injection, the deformable glass roof is pressed downwards by a convex lens, gently herding the biomolecules into the wells. The floor-ceiling distance can be controlled by pillars/posts attached to the chamber ceiling to leave a small gap by which to introduce molecules/reagents to the large, trapped biomolecules. The wells are deep enough to contain freely diffusing and fluctuating biomolecules, but shallow enough to keep them in the field of view for extended observation times. By squeezing out extra molecules, background fluorescence is reduced up to 20-fold and samples can be tested at higher concentrations compared to typical singlemolecule techniques. Furthermore, the array of embedded wells allows data collection with significant statistics within a single experiment.

Confinement microscopy enables a new approach to explore macromolecular behavior, specifically protein phase separation, on various dimensions under spatial confinement relevant to the cellular interior. We visualized formation of condensates and controlled their growth by systematically varying confinement dimensions (Fig. 2 and Fig. 3). We then explored the mesoscale properties of confined condensates by tracking the fluctuating motions of encapsulated fluorescent probe particles within the condensates. We investigated two liquid condensate systems. First, phase-separated polyethylene glycol (PEG) and dextran condensates were used to explore assembly, growth and final size under confinement. PEG-dextran condensates have been previously formed inside large vesicles, a conventional technique, as a simple model of the crowded cytoplasm (25-28). Second, we investigated biopolymer condensates of ribonucleoprotein complexes formed of polyuridine (polyU) RNA and protein Dhh1 as a simple, few-component model of natural NMOs. In both cases, we explored probe particle diffusion with respect to condensate size.

PEG-dextran mixtures exhibit anomalous diffusion and sizedependent properties: In the first system, fluorescentlylabeled dextran was confined within wells, and condensate formation and growth were monitored as PEG was introduced. The condensates formed under confinement (see Supplementary info S1 for Materials and Experimental Methods) were demonstrably smaller and more monodisperse than those formed on a featureless coverslip where condensate fusion may occur more quickly (Fig. 1c and Fig. 2a). When the reconstituent elements are confined in sealed wells, the maximum size of the phase separated condensate is controlled and limited by the amount of material available inside the well, i.e. proportional to the well size. For example, Fig. 2 compares condensates formed on a coverslip and within a large array of wells with a controlled final size of the condensates.

Previous work indicated that smaller condensates may have different network structure and physical behaviors compared to larger ones such that some mechanical theories would fail to explain their observed behavior $(29,30)$. To test whether the properties of confined condensates change with their size, we performed passive microrheology experiments. The motions of probe particles (20-nm fluorescent latex microspheres passivated with dextran), randomly distributed within condensates, were tracked using our in-house tracking algorithm (31) (Fig. 2b; Supplementary Info S2). To characterize diffusion properties of the embedded probe particles, we calculated their time-averaged mean squared displacements (MSD) (5), and performed correlation analyses on the videos of particle diffusion. Correlation methods such as fluorescence correlation spectroscopy $(32,33)$ and image correlation spectroscopy (34) are complimentary to single particle tracking, but are more objective in that individual point spread functions do not need to be fit. As such, correlation techniques are able to quantify faster diffusion coefficients or assess data with lower signal to noise ratios (35). The self-similarity of the fluorescent intensity signal at the pixel with the highest intensity fluctuations within the condensate was analyzed by second-order correlation (see Supplementary Info S3 for more details).

For solutions of pure dextran prior to PEG introduction, a homogeneous diffusion of beads was obtained in the microscale (Supplementary Figure S1). Interestingly, after PEG was introduced and phase separation occurred, dextran-rich condensates possessed heterogeneous properties (Fig. 4(a)). Probe particle diffusion varied from one condensate to another, and even from one location to another within the same condensate; MSD values varied by up to one order of magnitude.

To help elucidate the diffusion mechanisms within the PEGdextran condensates, we consider the concentration regimes of the system. Table 1 shows the theoretically calculated overlap concentration $\left(C^{*}\right)$, above which polymer chains start to interact and become an entangled network, and the estimation of the dextran concentration within a condensate $\left(\mathrm{C}_{\text {condensate }}\right)$. Overlap concentration is calculated based on $C^{*} \approx \frac{3 M_{w}}{4 \pi N_{A} R_{g}^{3}}$, where $M_{w}$ is molecular weight of the polymer (500 kDa for dextran and $57.5 \mathrm{kDa}$ for Dhh1), $N_{A}$ is Avogadro's number, and $R_{g}$ is radius of gyration of the polymer $(\sim 20 \mathrm{~nm}$ for dextran and $\sim 4.8 \mathrm{~nm}$ for Dhh1). Comparison suggests that a dextran-rich condensate is indeed a crowded, highly entangled system $\left(\mathrm{C}_{\text {condensate }}>\mathrm{C}^{*}\right)$. Crowded environments created by long chains of dextran molecules $(500 \mathrm{kDa})$ within a condensate may result in internal structural heterogeneity, leading to the observed heterogeneous diffusion behavior.

Particle motion is also affected by the confining surface (i.e. condensate-solvent interface) through solvent-mediated hydrodynamic interactions (36). As a particle moves inside a condensate, it propagates perturbations in the velocity field of the medium traveling back and forth between the interface and the particle. In a crowded environment, hydrodynamic interactions become even more complex and can create heterogeneity in particle mobility within a condensate.

PEG-dextran condensate properties appear to be sizedependent. Despite heterogeneity, ensemble averaged MSD curves show reduced mobility of probe particles inside smaller (1-3 $\mu \mathrm{m})$ condensates compared to inside larger (>3$15 \mu \mathrm{m}$ ) ones (Fig. 4 (a)). Correlation analysis (Supplementary Info S3) further supports the size dependence and heterogeneous diffusive motion of beads inside dextran conden- 
sates (Fig. 4(c)). According to the correlation analysis, particles have slower diffusion in the smaller condensates (see Table S1), similar to the results obtained from single particle tracking analysis.

Ribonucleo poly $(U)$-Dhhl condensates also exhibit anomalous diffusion: The next condensates we studied were the simple model NMOs formed from the yeast RNA binding protein Dhh1 and the RNA analogue poly(U). Here, we premixed the Dhh1 with the poly(U), and visualized formation of Dhh1-poly(U) condensates with controlled sizes using confinement microscopy (Fig. 3).

As in the PEG-dextran system, both particle tracking and correlation analysis (Fig. 5) indicated heterogeneous anomalous diffusion of embedded probe particles (48-nm fluorescent latex microspheres passivated with bovine serum albumin, BSA). However, unlike for the PEG-dextran condensates, the Dhh1-poly(U) condensates did not exhibit significant size-dependent internal properties. In addition, the averaged correlation curves were better fit with a two-component correlation function, i.e. two diffusion components (Fig. 5c and Supplementary Information S3), suggesting both a slow and a fast mode of diffusion. The faster component had an anomalous factor, $\alpha,>1$ indicative of a super-diffusive, hopping mechanism.

Anomalous diffusion and hopping mechanisms have recently been reported in vivo in stress granules, a different type of NMO (37). In that case, biphasic partitioning of biopolymers resulted in their having suppressed diffusion in some local microenvironments but larger mobility at other locations (38).

Single-component Brownian curves failed to fit the data (Supplementary Figure S2). Similar to the single-particle tracking results, there were statistically similar values for the $\operatorname{Dhh1-poly(U)~condensates~regardless~of~size~(Supplemen-~}$ tary Table S1).

In order to better understand the anomalous (non-Brownian) diffusion within condensates, we measured the displacement probability distribution. Compared to a Gaussian model, there are too many large displacement events (the tails in Fig. 5(b) at high and low $\Delta x$ values) and a stronger confinement of motion (the sharp peak close to zero displacement, $\Delta x=0$ ). These observations provide further evidence for the "hopping diffusion" mechanism where a particle mostly resides in a caged structure made by neighboring molecules but occasionally escapes and moves a significantly larger distance than a normal Brownian diffusion would predict $(38,39)$. The non-Gaussian distribution of particle displacement was also observed in dextran condensates (Fig. 4(b)) with tails at long displacements and is an indication of a heterogeneous environment, although unlike in protein condensates, hopping diffusion is not directly observed from analysis of particle trajectory (inset in Fig. 4b).

To explain the diffusion mechanisms within the protein condensates, protein concentrations inside condensates were estimated. The estimated Dhh1 concentration (see Supplementary Figure S3) inside a condensate and the theoretical overlap concentration for the protein condensates are shown in
Table 1. Comparison of these values suggests that Dhh1 protein itself does not create a significantly crowded condensate interior, yet we still observe confined motion of embedded probe particles. Poly(U) molecules may be unevenly distributed within the condensate to cause locally crowded environments that inhibit particle motion. It is also possible that specific interactions between the condensate components contribute to the condensate interior properties and effectively suppress particle movement. Binding of ATP and RNA to Dhh1 has been previously shown to be necessary for condensate formation, presumably due to inducing specific conformations of the Dhh1 protein (6). A schematic of this bound conformation of poly $(\mathrm{U})$ and $\mathrm{Dhh} 1$ is shown in Fig. 3(d).

To explore the effect of Dhh1-/poly(U) bound conformation in condensate assembly, we introduced the regulatory protein Not1 to the confined condensates. Not1 enhances the ATPase activity of Dhh1, unbinds Dhh1 from the nucleic acid by changing the Dhh1 conformation, and ultimately results in disassembly of the condensate (6). Interestingly, we observed that when we added Not1, embedded beads started to diffuse more quickly and eventually diffused freely throughout the pit in the solution of the poly(U) and Dhh1 (Supplementary Figure S4). Our particle tracking measurements showed that in an ATP- and RNA-bound Dhh1 conformation, i.e. assembled condensate, probe diffusion was highly suppressed and non-homogeneous across the condensate, but as the Dhh1 conformation changed due to the presence of Not1, probe particles diffused more freely and uniformly.

Other results have indicated that permeability and diffusion inside a protein condensate are affected by the length (MW) of the component RNA (40). These specific effects will be dictated by the type of RNA- protein interactions. Short RNAs may modulate protein-protein interactions to lead to a lowering of the viscosity, while larger molecules have the opposite effect (41), perhaps because larger molecules are involved in additional physical processes such as entanglement. In our protein study, the model RNA molecule, poly(U), is polydisperse in size $(100 \mathrm{kDa}<\mathrm{MW}<1000 \mathrm{kDa})$, making it potentially more biologically representative than a monodisperse RNA molecule. This polydispersity likely enhances the heterogeneity of the condensate interior structure, as indicated by our observed particle diffusion.

In summary, this study showcases a new application of confinement microscopy, which has enabled 1) control of condensate size through spatial confinement (i.e. well with a particular size), as shown in Fig. 3(a-c), and 2) the quantitative characterization of micro-environments within condensates. We obtained heterogeneous dynamics as well as anomalous diffusion inside both dextran-rich and protein-rich condensates. In ribonucleo protein-rich condensates, we found evidence of temporal heterogeneity such that embedded particles may diffuse within a microenvironment and occasionally "hop" to a neighboring microenvironment with different physical properties. Furthermore, we observed a size dependence of diffusion, and thus interior condensate properties, for dextran condensates, although this was not observed sig- 
nificantly for the Dhh1-poly(U) condensates tested.

The presence of microenvironments inside NMO condensates has important biological implications. Proposed to arise in part due to heterogeneous distribution of nucleic acids within a condensate, microenvironments likely facilitate biochemical processes such as gene expression (42). Molecular crowding also contributes to the stochasticity of biochemical processes within NMOs by decreasing diffusion of proteins and nucleic acids and contributing to the uneven distribution of biomolecules (42). In vitro studies at in vivo size scales should shed light on important condensate properties. Confinement microscopy is a promising technique for a variety of NMO investigations, providing insight into single molecule dynamics and reaction kinetics, by creating "cell-like" conditions of spatial confinement and crowding.

\section{Acknowledgements}

M.S., S.W.M, and S.R.L. acknowledge support from the Canadian Institutes of Health Research (CIHR) grants MOPGMX-152556 (S.W.M.), the Human Frontier Science Program RGP0034/2017 (S.W.M.), the Natural Sciences and Engineering Research Council grant, Discovery and Accelerator Grant Programs (S.R.L.), and CREATE Program on the Cellular Dynamics of Macromolecular Complexes (M.S.). L.K. thanks the Arnold O. and Mabel M. Beckman Foundation for support by the Beckman-Brown Interdisciplinary Postdoctoral Fellowship. The authors thank Karsten Weis and Marie Hondele (ETH-Zurich) for gifts of Dhh1 and Not1 proteins and also thank Christine Desroches for supporting protein condensation experimental design.

\section{Conflict of interest}

There are no conflicts to declare.

\section{Bibliography}

1. Salman F. B Banani, Hyun O. Lee, Anthony A. Hyman, and Michael K. Rosen. Biomolecular condensates: organizers of cellular biochemistry. Nature Reviews Molecular Cell Biology, 18:285-298, 2017.

2. Yongdae Shin and Clifford P. Brangwynne. Liquid phase condensation in cell physiology and disease. Science, 357(6357), 2017. ISSN 0036-8075. doi: 10.1126/science.aaf4382.

3. Louis-Philippe Bergeron-Sandoval, Nozhat Safaee, and Stephen W Michnick. Mechanisms and consequences of macromolecular phase separation. Cell, 165(5):1067-1079, 2016.

4. Clifford P Brangwynne. Phase transitions and size scaling of membrane-less organelles. $J$ Cell Biol, 203(6):875-881, 2013

5. Marina Feric, Nilesh Vaidya, Tyler S Harmon, Diana M Mitrea, Lian Zhu, Tiffany M Richardson, Richard W Kriwacki, Rohit V Pappu, and Clifford P Brangwynne. Coexisting liquid phases underlie nucleolar subcompartments. Cell, 165(7):1686-1697, 2016.

6. Christopher Frederick Mugler, Maria Hondele, Stephanie Heinrich, Ruchika Sachdev, Pascal Vallotton, Adriana Y Koek, Leon Y Chan, and Karsten Weis. Atpase activity of the dead-box protein dhh1 controls processing body formation. Elife, 5:e18746, 2016.

7. Wylie Stroberg and Santiago Schnell. On the origin of non-membrane-bound organelles, and their physiological function. Journal of Theoretical Biology, 434:42-49.

8. Ana Eulalio, Isabelle Behm-Ansmant, and Elisa Izaurralde. P bodies: at the crossroads of post-transcriptional pathways. Nature reviews. Molecular cell biology, 8(1):9, 2007.

9. Avinash Patel, Hyun $O$ Lee, Louise Jawerth, Shovamayee Maharana, Marcus Jahnel, Marco Y Hein, Stoyno Stoynov, Julia Mahamid, Shambaditya Saha, Titus M Franzmann, et al. A liquid-to-solid phase transition of the als protein fus accelerated by disease mutation. Cell, 162(5):1066-1077, 2015.

10. J Ross Buchan. mrnp granules: assembly, function, and connections with disease. RNA biology, 11(8):1019-1030, 2014.

11. Paul Anderson, Nancy Kedersha, and Pavel Ivanov. Stress granules, $p$-bodies and cancer. Biochimica et Biophysica Acta (BBA)-Gene Regulatory Mechanisms, 1849(7):861-870, 2015.

12. Jeffrey A Toretsky and Peter E Wright. Assemblages: functional units formed by cellular phase separation. J Cell Biol, 206(5):579-588, 2014.
13. Stephanie $C$ Weber and Clifford $P$ Brangwynne. Inverse size scaling of the nucleolus by a concentration-dependent phase transition. Current Biology, 25(5):641-646, 2015.

14. Xiaolong Yang and Tian Xu. Molecular mechanism of size control in development and human diseases. Cell research, 21(5):715, 2011.

15. Stefan Schütz, Erik R Nöldeke, and Remco Sprangers. A synergistic network of interactions promotes the formation of in vitro processing bodies and protects mrna against decapping. Nucleic Acids Research, 45(11):6911-6922, 2017.

16. Diana M. Mitrea and Richard W. Kriwacki. Phase separation in biology; functional organization of a higher order. Cell Communications and Signaling, 14(1):1-20, 2016

17. William M. Aumiller Jr. and Christine D. Keating. Experimental models for dynamic compartmentalization of biomolecules in liquid organelles: Reversible formation and partitioning in aqueous biphasic systems. Advances in Colloid and Interface Science, 239:75 - 87, 2017. ISSN 0001-8686.

18. Vladimir N Uversky. Intrinsically disordered proteins in overcrowded milieu: membrane-less organelles, phase separation, and intrinsic disorder. Current opinion in structural biology, 44:18-30, 2017.

19. Lian Zhu and Clifford P Brangwynne. Nuclear bodies: the emerging biophysics of nucleoplasmic phases. Current opinion in cell biology, 34:23-30, 2015.

20. Yang Luo, Zhenkun $\mathrm{Na}$, and Sarah A. Slavoff. P-bodies: Composition, properties, and functions. Biochemistry, $0(0)$ :null, 0 .

21. Daniel J Berard, François Michaud, Sara Mahshid, Mohammed Jalal Ahamed, Christopher MJ McFaul, Jason S Leith, Pierre Bérubé, Rob Sladek, Walter Reisner, and Sabrina R Leslie. Convex lens-induced nanoscale templating. Proceedings of the National Academy of Sciences, 111(37):13295-13300, 2014

22. Mohammed Jalal Ahmed, Sara Mahshid, Daniel J Berard, François Michaud, Rob Sladek, Walter W Reisner, and Sabrina R Leslie. Continuous confinement fluidics: Getting lots of molecules into small spaces with high fidelity. Macromolecules, 49(7):2853-2859, 2016.

23. Gil Henkin, Daniel Berard, Francis Stabile, Marjan Shayegan, Jason S Leith, and Sabrina R Leslie. Manipulating and visualizing molecular interactions in customized nanoscale spaces. Analytical chemistry, 88(22):11100-11107, 2016.

24. Daniel J Berard, Marjan Shayegan, Francois Michaud, Gil Henkin, Shane Scott, and Sabrina Leslie. Formatting and ligating biopolymers using adjustable nanoconfinement. Applied Physics Letters, 109(3):033702, 2016.

25. Yanhong Li, Reinhard Lipowsky, and Rumiana Dimova. Membrane nanotubes induced by aqueous phase separation and stabilized by spontaneous curvature. Proceedings of the National Academy of Sciences, 108(12):4731-4736, 2011.

26. Yanhong Li, Reinhard Lipowsky, and Rumiana Dimova. Transition from complete to partial wetting within membrane compartments. J. Am. Chem. Soc., 130(37):12252-12253, 2008.

27. Marcus R. Helfrich, Lauren K. Mangeney-Slavin, M. Scott Long, Karrera Y. Djoko, and Christine D. Keating. Aqueous phase separation in giant vesicles. J. Am. Chem. Soc., 124(45): 13374-13375, 2002

28. Takahiko Ban, Takashi Fukuyama, Shouta Makino, Erika Nawa, and Yuichiro Nagatsu. Selfpropelled vesicles induced by the mixing of two polymeric aqueous solutions through a vesicle membrane far from equilibrium. Langmuir, 32(11):2574-2581, 2016.

29. Robert W. Style, Callen Hyland, Rostislav Boltyanskiy, Wettlaufer John S., and Eric R. Dufresne. Surface tension and contact with soft elastic solids. Nature Communications, 4:1-6, 2013.

30. M. M. A. E. Claessens, R. Tharmann, K. Kroy, and A. R. Bausch. Microstructure and viscoelasticity of confined semiflexible polymer networks. Nature Physics, 2:186-189, 2006.

31. Shane Scott, ZhiMing Xu, Fedor Kouzine, Daniel J Berard, Cynthia Shaheen, Barbara Gravel, Laura Saunders, Alexander Hofkirchner, Catherine Leroux, Jill Laurin, David Levens, Craig J Benham, and Sabrina R Leslie. Visualizing structure-mediated interactions in supercoiled dna molecules. Nucleic Acids Research, page gky266, 2018. doi: 10.1093/nar/gky266.

32. Elliot L. Elson and Douglas Magde. Fluorescence correlation spectroscopy. i. conceptual basis and theory. Biopolymers, 13(1):1-27, 1974

33. P. Schwille and J. Ries. Principles and applications of fluorescence correlation spectroscopy (fcs). pages 63-85, 2011.

34. Justin T. Cooper and Joel M. Harris. Imaging fluorescence-correlation spectroscopy for measuring fast surface diffusion at liquid/solid interfaces. Analytical Chemistry, 86(15): 7618-7626, 2014. doi: 10.1021/ac5014354. PMID: 24975169.

35. Lydia Kisley, Rachel Brunetti, Lawrence J. Tauzin, Bo Shuang, Xiyu Yi, Alec W. Kirkeminde, Daniel A. Higgins, Shimon Weiss, and Christy F. Landes. Characterization of porous materials by fluorescence correlation spectroscopy super-resolution optical fluctuation imaging. ACS Nano, 9(9):9158-9166, 2015. doi: 10.1021/acsnano.5b03430. PMID: 26235127.

36. A. E. Cervantes-Martínez, A. Ramírez-Saito, R. Armenta-Calderón, M. A. Ojeda-López, and J. L. Arauz-Lara. Colloidal diffusion inside a spherical cell. Phys. Rev. E, 83:030402, Mar 2011. doi: 10.1103/PhysRevE.83.030402.

37. Benedikt Niewidok, Maxim Igaev, Abel Pereira da Graca, Andre Strassner, Christine Lenzen, Christian P. Richter, Jacob Piehler, Rainer Kurre, and Roland Brandt. Singlemolecule imaging reveals dynamic biphasic partition of rna-binding proteins in stress granules. The Journal of Cell Biology, 2018. doi: 10.1083/jcb.201709007.

38. Michael J. Skaug, Joshua Mabry, and Daniel K. Schwartz. Intermittent molecular hopping at the solid-liquid interface. Phys. Rev. Lett., 110:256101, Jun 2013. doi: 10.1103/PhysRevLett. 110.256101 .

39. M. T. Valentine, P. D. Kaplan, D. Thota, J. C. Crocker, T. Gisler, R. K. Prud'homme, M. Beck, and D. A. Weitz. Investigating the microenvironments of inhomogeneous soft materials with multiple particle tracking. Phys. Rev. E, 64:061506, Nov 2001. doi: 10.1103/PhysRevE.64. 061506.

40. Ming-Tzo Wei, Shana Elbaum-Garfinkle, Alex S Holehouse, Carlos Chih-Hsiung Chen, Marina Feric, Craig B Arnold, Rodney D Priestley, Rohit V Pappu, and Clifford P Brangwynne. Phase behaviour of disordered proteins underlying low density and high permeability of liquid organelles. Nature Chemistry, 9:1118, jun 2017.

41. Shana Elbaum-Garfinkle, Younghoon Kim, Krzysztof Szczepaniak, Carlos Chih-Hsiung 
bioRxiv preprint doi: https://doi.org/10.1101/402230; this version posted August 28,2018 . The copyright holder for this preprint (which was not certified by peer review) is the author/funder. All rights reserved. No reuse allowed without permission.

Chen, Christian R. Eckmann, Sua Myong, and Clifford P. Brangwynne. The disordered $\mathrm{p}$ granule protein laf-1 drives phase separation into droplets with tunable viscosity and dynamics. Proceedings of the National Academy of Sciences, 112(23):7189-7194, 2015. ISSN 0027-8424. doi: 10.1073/pnas.1504822112.

42. Maike M. K. Hansen, Lenny H. H. Meijer, Evan Spruijt, Roel J. M. Maas, Marta Ventosa Rosquelles, Joost Groen, Hans A. Heus, and Wilhelm T. S. Huck. Macromolecular crowding creates heterogeneous environments of gene expression in picolitre droplets. $\mathrm{Na}$ ture Nanotechnology, 11:191--197, 2016. 
bioRxiv preprint doi: https://doi.org/10.1101/402230; this version posted August 28, 2018. The copyright holder for this preprint (which was not certified by peer review) is the author/funder. All rights reserved. No reuse allowed without permission.
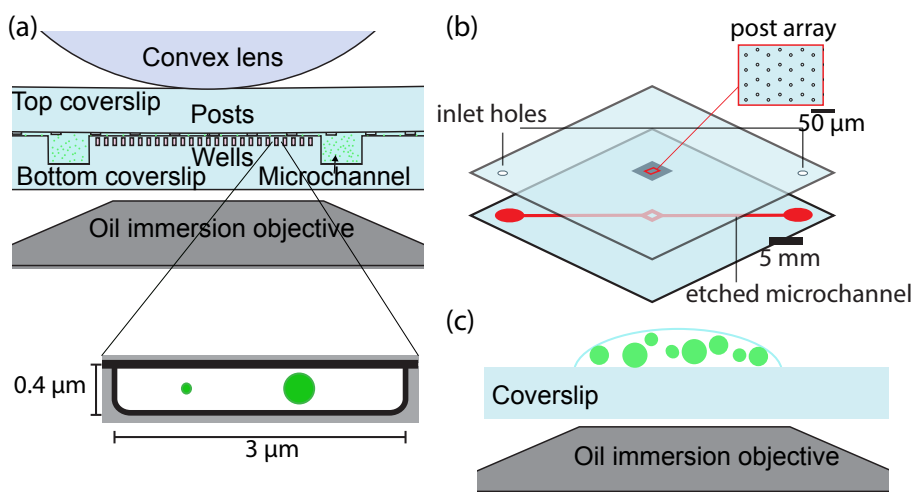

Fig. 1. Confinement microscopy: (a) schematic of a Convex Lens-induced Confinement (CLiC) instrument used here; a convex lens deforms the top coverslip of the flow cell. Posts fabricated into the top coverslip maintain a space above the wells ( $450 \mathrm{~nm}$ deep and $3 \mu \mathrm{m}$ in diameter) embedded in the flow cell floor, where condensates are formed).(b) Schematic of a microchannel fabricated into the bottom coverslip that encircles the embedded pit array and facilitates reagent addition and buffer exchange. (c) Schematic showing condensate formation on an open surface, i.e. no confinement. 
bioRxiv preprint doi: https://doi.org/10.1101/402230; this version posted August 28,2018 . The copyright holder for this preprint (which was not certified by peer review) is the author/funder. All rights reserved. No reuse allowed without permission.
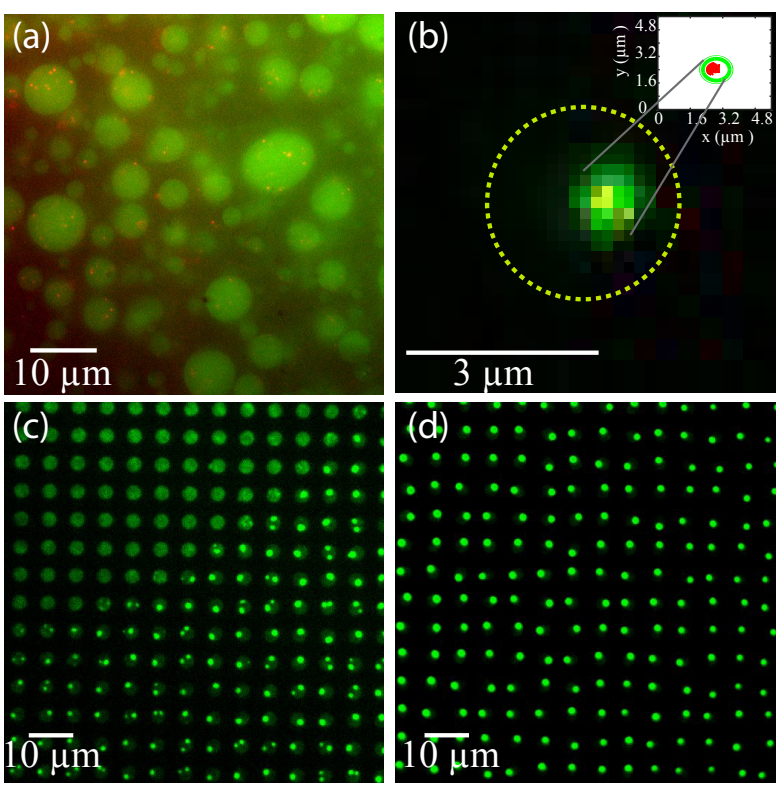

Fig. 2. Dextran-rich condensates condensed from a mixture of PEG-dextran formed (a) on an open surface (i.e. without confinement), and (b) inside a $3 \mu$ m well (i.e. under confinement). Using dual color imaging, fluorescently-labeled beads (red) are distinguished in dextran-rich condensates (green). Note: Red beads appear yellow in the green channel when they overlap with green-labeled dextran-rich droplets. Dashed circle: well location. Inset: the particle tracking of a red-labeled bead inside the condensate (green circle). (c) Initiation of phase separation in an array of wells containing confined labeled dextran, as PEG is introduced from the bottom right corner. (d) Same array as shown in (c) 5 minutes later, condensates reach their final size. 
bioRxiv preprint doi: https://doi.org/10.1101/402230; this version posted August 28,2018 . The copyright holder for this preprint (which was not certified by peer review) is the author/funder. All rights reserved. No reuse allowed without permission.
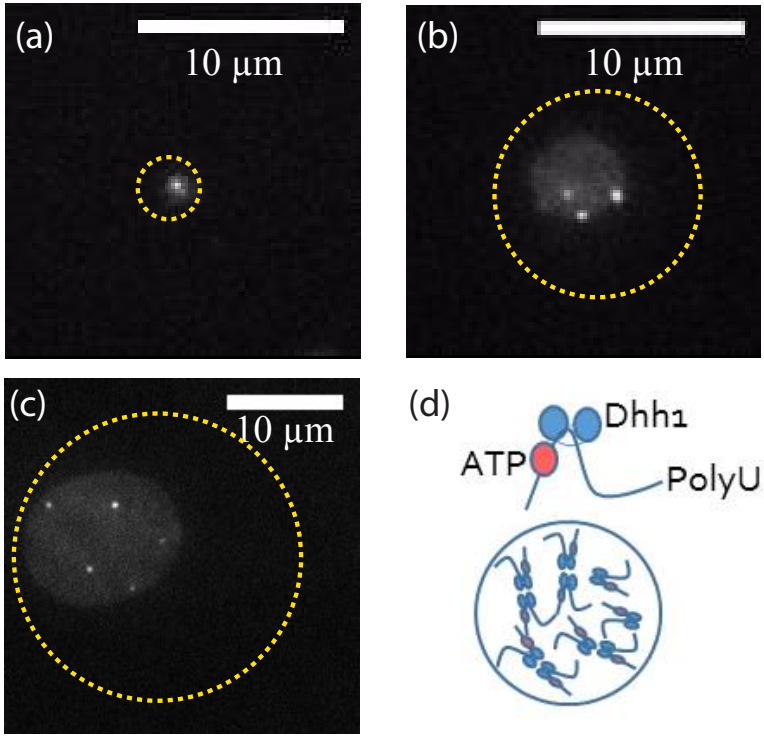

(d)

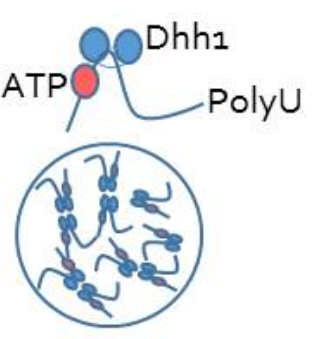

Fig. 3. Effect of confinement on the final size of the protein condensates: condensate of (a) $2 \mu \mathrm{m}$, (b) $4 \mu \mathrm{m}$, and (c) $14 \mu \mathrm{m}$ formed in wells with diameters of 3 , 10 , and 25 $\mu \mathrm{m}$, respectively. Dashed circles correspond to the location of the wells. Condensates are shown in gray and bright dots inside condensates are probing particles of $48 \mathrm{~nm}$ in diameter. (d) Schematic of ATP- and RNA-bound Dhh1 conformation and how these components may interact with each other inside a phase separated condensate. 
bioRxiv preprint doi: https://doi.org/10.1101/402230; this version posted August 28,2018 . The copyright holder for this preprint (which was not certified by peer review) is the author/funder. All rights reserved. No reuse allowed without permission.
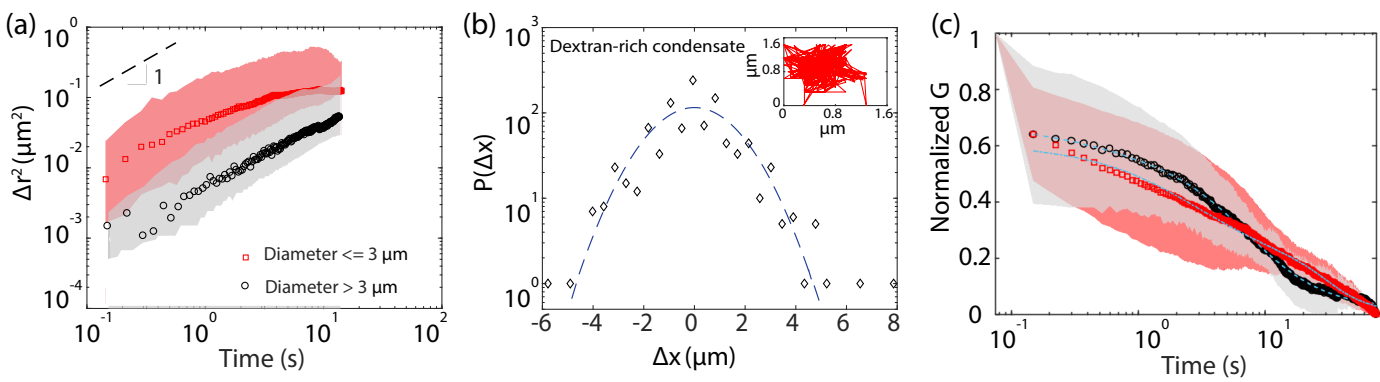

Fig. 4. Diffusion analysis for probe particles inside PEG-dextran condensates $(\mathrm{N}=135)$ : Red and black represent condensates that are smaller and larger than $3 \mu \mathrm{m}$, respectively. (a) Time-averaged mean squared displacement versus lag time of probe particles embedded in dextran-rich condensates. For each data set, the circles or squares show the averaged MSD (i.e. time and ensemble averaged), and the shaded area the distribution of MSD curves within that set. Dashed line: a slope of 1 in log-log scale, indicative of normal Brownian diffusion. (b) Typical displacement probability distribution, measured for a probe particle embedded inside a dextran-rich condensate, plotted logarithmically against displacement. The dashed curve represents a fitted standard Gaussian distribution, and highlights the poor fit to Brownian solutions with the experimental data. Inset: probe particle trajectory over 1 minute. (c) Correlation analysis performed on probe particle diffusion inside a dextran-rich condensates. Circles or squares show the averaged correlation curve, $\mathrm{G}$, for each data set, while the shaded areas show the distribution of correlation curves. Lines represent the fit which give two decay times (relevant to a slow and a fast diffusion mode) and power law exponents. 
bioRxiv preprint doi: https://doi.org/10.1101/402230; this version posted August 28,2018 . The copyright holder for this preprint (which was not certified by peer review) is the author/funder. All rights reserved. No reuse allowed without permission.
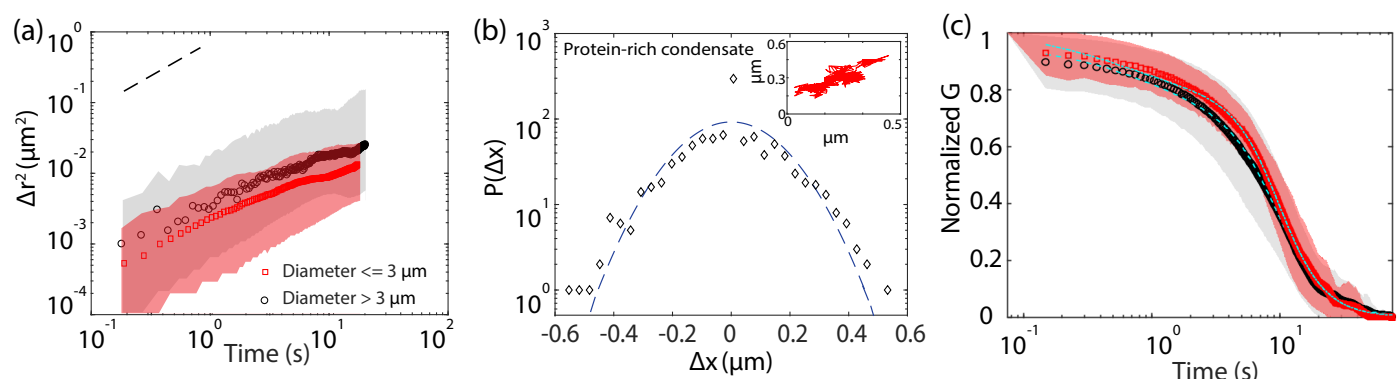

Fig. 5. Diffusion analysis for probe particles inside protein-nucleic acid condensates ( $N=140)$ : Red and black represent condensates that are smaller and larger than $3 \mu \mathrm{m}$, respectively. (a) Time-averaged mean squared displacement versus lag time of probe particles embedded in protein-rich condensates. (b) Typical displacement probability distribution, measured for a probe particle embedded inside a protein-rich condensate plotted logarithmically against displacement. The non-Gaussianity observed can be attributed to the hopping diffusion. Inset: the trajectory of a probe particle over 1 minute. It suggests that the particle spends most of its time in a cage formed by its neighboring chain structures while during rare events moves a significant distance due to a rearrangement of cage structure. (c) Correlation analysis performed on probe particles diffusing inside a protein-rich condensate. 
bioRxiv preprint doi: https://doi.org/10.1101/402230; this version posted August 28,2018 . The copyright holder for this preprint (which was not certified by peer review) is the author/funder. All rights reserved. No reuse allowed without permission.

Table 1. Comparison of overlap concentration with polymer concentration within a condensate for a dextran-rich condensate and Dhh1-rich condensate

\begin{tabular}{lllll}
\hline Concentration & $\mathrm{C}^{*}$ & $\mathrm{C}_{\text {initial }}$ & $\mathrm{C}_{\text {condensate }}$ & Comparison \\
\hline Dextran $(\mathrm{wt} \%)$ & 1.8 & 2 & $>2$ & $\mathrm{C}_{\text {condensate }}>\mathrm{C}^{*}$ \\
Dhh1 $(\mathrm{mM})$ & 3.5 & 0.012 & 0.125 & $\mathrm{C}_{\text {condensate }}<\mathrm{C}^{*}$ \\
\hline
\end{tabular}

